\title{
Admission avoidance using intramuscular antibiotics for the treatment of borderline foot infections in people with diabetes in a tertiary care foot clinic
}

\author{
Ketan Dhatariya
}

\begin{abstract}
Several international guidelines exist to help decision making for the infected 'diabetic foot'. However, none consider admissions avoidance. We wanted to develop an antibiotic foot formulary for the empirical treatment of diabetes related foot infections presenting to our service and subsequently to asses the costs associated with the introduction of our protocol. We rationalised our antibiotic protocol. The introduction of our formulary changed the average antibiotic prescribing costs for a 3 week course of treatment from £17.12 to £16.42. In addition, we adapted the Infectious Disease Society of America (IDSA) guideline by introducing a category of 'moderate infection - borderline admission' to our classification. This enabled the administration of outpatient intramuscular antibiotics. Over 22 months of follow up, 26 episodes were eligible for treatment with intramuscular antibiotics. Over the same time period, 121 people were admitted directly from the foot clinic. The costs saved as a result of avoided or delayed admission for those 26 episodes was over $£ 76,000$. For 12 people who required subsequent admission, their length of hospital stay was significantly shorter than those admitted directly (9.25 days [range 2-25] vs. 16.11 [2-64] p=0.045). In summary, by modifying the IDSA classification and adopting a protocol to administer outpatient oral and intramuscular antibiotics we have led to substantial cost savings, shorter hospital admissions and also have developed a successful admissions avoidance strategy.
\end{abstract}

\section{Problem}

Foot infection in people with diabetes is a very common complication, with previous work showing that up to $58 \%$ of diabetes related foot ulcers were infected (1). Foot infections remain one of the commonest diabetes related cause of acute hospital admission (2). We wanted to try and reduce the number of admissions from our specialist multidisciplinary diabetic foot clinic by developing our own diabetic foot antibiotic protocol.

\section{Background}

To date, the choice of antibiotic regimen for use in diabetes related foot infections has largely remained at the discretion of the prescribing physician. Whilst deep tissue samples or swab cultures are key to guiding antibiotic choice and should always be sought when treatment is being considered, most often, only superficial specimens are taken.

Empirical therapy using narrow spectrum antibiotics active against aerobic gram positive cocci are the most commonly prescribed agents because these are the predominant microorganisms that colonise and infect ulcers $(3,4)$. Broad spectrum empirical therapy is only indicated for severe infections and for infections in ischaemic feet (4). It has also been recommended that the choice of this empirical antibiotic therapy and the route of its administration should be determined by the severity of the infection and the likely aetiological organisms (4). The UK National Institute for Health and Clinical Excellence (NICE) added the caveat that the antibiotic with the lowest acquisition costs be used (5).
In 2008 the reconfiguration of our specialist multidisciplinary diabetic foot service included the recognition that we needed a more cohesive approach to empirical antibiotic prescribing. We reviewed several guidelines and felt that the Infectious Disease Society of America's (IDSA) guideline, whilst excellent and widely used, was limiting because it relied mainly on the use of oral antibiotics in the outpatient setting for those who did not need hospitalisation, or intravenous antibiotics for those who did. We felt there was a category of patients for whom their infections were too severe for oral antibiotics alone, but for whom hospitalisation was potentially avoidable. We termed this degree of infection as 'moderate infection - borderline admission'.

We introduced our guideline and did a cost analysis of the rationalisation of agents, as well as an economic analysis of admissions avoided as a result of the use of intramuscular agents.

\section{Baseline measurement}

Of the 144 sets of note available for review, of the 288 who fulfilled the criteria, 64 were from patients given antibiotics prior to the introduction of our protocol and the remaining 80 were from after its introduction.

The most commonly prescribed regimen prior to the introduction of the protocol was the combination of amoxicillin $500 \mathrm{mg}$ three times daily and flucloxacillin $500 \mathrm{mg}$ four times daily. The second most commonly prescribed regimen was amoxicillin 500mg three times daily, flucloxacillin $500 \mathrm{mg}$ four times daily, and metronidazole $400 \mathrm{mg}$ three times daily. The cheapest regimen prescribed was 
erythromycin $500 \mathrm{mg}$ four times daily. The costs per patient for an average three week course of these regimens were $£ 16.50, £ 20.85$ and $£ 12.24$ respectively.

The overall average cost per patient for an average three week cost on any of the pre-protocol regimes was $£ 17.12$ per patient.

See supplementary file: ds1612.doc - "Table 1"

\section{Design}

The antibiotic foot policy that developed by the multidiscilplinary foot team is shown in Table 1. Our team comprises of diabetologists, podiatrists, specialist antimicrobial pharmacists, vascualr and orthopaedic surgeons and microbiologists

\section{Strategy}

The number of prescribers in the foot clinic was limited to those who ran foot clinics, and the guideline was widely advertised within our Trust. The guideline was ratified by the Drugs, Therapeutics and Medicines Management Committee of our institution, as well as the Therapeutic Advisory Group of our Primary Care Trust. A copy of the guideline was hosted on the county wide General Practice electronic register.

\section{Results}

We analysed the notes of the 80 patients treated after the introduction of the protocol who fulfilled the inclusion criteria of new lesions with no previous antibiotic use. The most commonly prescribed regimen was co-amoxiclav $625 \mathrm{mg}$ three times daily with an estimated cost of $£ 16.29$ per patient for a three week course.

Between January 2009 and October 2010 we also prescribed intramuscular ceftriaxone together with oral ciprofloxacin and metronidazole 26 times in 23 individual patients, all of whom were treated according to our guideline. The daily cost of the intramuscular and oral antibiotic regimen (ceftriaxone $1 \mathrm{~g}$ in $3.5 \mathrm{mls}$ of $1 \%$ lidocaine, oral ciprofloxacin $500 \mathrm{mg}$ twice daily and oral metronidazole $400 \mathrm{mg}$ three times daily) based on the 2010 British National Formulary prices was $£ 10.34$ per day (6). The total cost of the intramuscular and oral antibiotics used for those 14 episodes in whom admission was avoided was $£ 6,633.48$.

The assumption made was that prior to the introduction of our protocol, these individuals would have been admitted for intravenous antibiotics. If they were to have stayed for the same average length of time that those who were admitted directly stayed, i.e. 16.11 days, this equated to a saving of 225.54 bed days. In 2010 our institution estimated a 24 hour stay in a hospital bed was £274. Thus, avoiding admission for those 14 episodes led to a saving of $£ 61,797.96$. However, given that the expenditure on antibiotics given in the community was $£ 6,633.48$, the actual estimated saving was therefore $£ 55,164.48$ or $£ 3,940.32$ per patient per episode.
Furthermore, looking at the outcomes for the 12 patients who had to be admitted despite having been treated with intramuscular and oral antibiotics as outpatients. These individuals were in hospital for an average of 9.25 days (range 2-25), i.e. 6.86 days less than those people who had been admitted directly from foot clinic. This led to a saving of 82.32 bed days (at $£ 274$ per day), making an estimated saving of $£ 22,550.68$ or $£ 1879.64$ per patient.

District (community) and practice nursing time was then factored into those people treated as outpatient. We estimated an hour per day at a cost of $£ 16$ per hour. Thus for the 26 people treated with intramuscular and oral antibiotics at a cost of $£ 10.74$ per day, we avoided or reduced hospital admission by 307.86 days ([14 $\times 16.11]$ $+[12 \times 6.86])$. The total thus spent on antibiotics and nurse time was $£ 3,306.42+£ 4,925.76=£ 8,232.18$. The costs avoided from hospital admission $£ 274 \times 307.86=£ 84,353.64$. This does not include the costs of intravenous antibiotics. Thus in these 26 episodes, we estimate a saving of $£ 76,121$.46. This is likely to be an underestimate.

\section{Lessons and limitations}

We have presented data to show that rationalising our empirical antibiotic protocol for the management of foot infections in people with diabetes has led to significant costs savings. Using a coordinated approach from all members of the specialist diabetic foot team, we got agreement from all of the major stake holders to ensure they understood the importance of the economic impact of diabetes related foot infections, and our attempt to tackle this.

There are some limitations to our data. We collected data on relatively small numbers of patients. Despite this, there are very few data in the literature that examine the use of intramuscular antibiotics, and thus we feel that to present this dataset may be valuable.

The data on costs has been estimated from those quoted in an the British National Formulary, and may not be applicable to the National Health Service in general because many hospitals may get 'discounts' on bulk purchases from suppliers. It may have been better to calculate them using the drug tariff. A further limitation may be due to the estimation of the length of the course of antibiotics. However, given that the costs using the protocol were marginally cheaper, it is likely that if prolonged courses were used, the cost savings would have been greater. Another limitation when discussing the use of intramuscular antibiotics was that those people who were admitted directly from our foot clinic had, by definition, more severe disease. Thus is may be assumed that because of this, they would have stayed in hospital longer than those who had a lesser degree of infection (albeit one that prior to the introduction of the protocol would also have led to admission). We also did not include those people who may have been admitted directly from other clinics - e.g. vascular or orthopaedic. However, members of our foot MDT communicate almost daily ensuring any patient with diabetes admitted to our institution with a diabetes related foot problem is seen by a member of the foot MDT within 24 hours of admission. 


\section{Conclusion}

We have introduced a new standardised initial empirical antibiotic policy that has modified the IDSA guideline. We found that by collaborating within the multidisciplinary diabetes foot clinic team we rationalised the prescribing of antimicrobials at no additional drug cost. This rationalisation has meant that the cost of treatment has stayed relatively unchanged. At the same time we have also simplified the regimes in an attempt to improve patient compliance. Our intramuscular regimen saved over $£ 60,000$ in just 23 patients over a period of 22 months. We believe that this is a cost effective strategy for admission avoidance.

\section{References}

1. Prompers L, Huijberts M, Schaper N, Apelqvist J, Bakker $\mathrm{K}$, Edmonds $\mathrm{M}$ et al. Resource utilisation and costs associated with the treatment of diabetic foot ulcers. Prospective data from the Eurodiale Study. Diabetologia 2008; 51(10):1826-1834.

2. Health and Social Care Information Centre. National diabetes inpatient audit 2011. https://catalogue.ic.nhs.uk/publications/clinical/diabetes/natidiab-inp-audi-11/nati-diab-inp-audi-11-nat-rep.pdf. 2012. Last accessed 8th April 2013

3. Lipsky BA, Pecoraro RE, Wheat LJ. The diabetic foot. Soft tissue and bone infection. Infect Dis Clin North Am 1990; 4(3):409-432.

4. Lipsky BA. A report from the international consensus on diagnosing and treating the infected diabetic foot. Diabetes Metab Res Rev 2004; 20(Suppl 1):S68-77.

5. National Institute for Clinical and Healthcare Excellence. Diabetic foot problems. Inpatient management of diabetic foot problems. CG119.

http://www.nice.org.uk/nicemedia/live/13416/53556/53556.p df. 2011. LAst accessed 8th April 2013

6. British Medical Association, Royal Pharmaceutical Society of Great Britain. British National Formulary. 60 ed. London: 2010.

\section{Declaration of interests}

Nothing to declare.

\section{Acknowledgements}

None 